\title{
PROGRESS
}

\section{Molecular mechanisms of complement evasion: learning from staphylococci and meningococci}

Davide Serruto, Rino Rappuoli, Maria Scarselli, Piet Gros and Jos A. G. van Strijp

Abstract | The complement system is a crucial component of the innate immune response in humans. Recent studies in Staphylococcus aureus and Neisseria meningitidis have revealed how these bacteria escape complement-mediated killing. In addition, new structural data have provided detailed insights into the molecular mechanisms of host defence mediated by the complement system and how bacterial proteins interfere with this process. This information is fundamental to our understanding of bacterial pathogenesis and may facilitate the design of bettervaccines.

To survive in the human host, bacteria have developed many different strategies to escape the innate immune response, including the expression of an extracellular capsule and 'hiding' within host cells, either in a vacuole or in the cytoplasm. Over the past few years it has become clear that, in addition, bacteria can escape recognition by the complement system through the actions of cell-surface or secreted proteins ${ }^{1,2}$.

Complement is one of the most important components of the innate immune system and is composed of more than 30 proteins that are present in human serum and tissue fluid as well as on cell surfaces ${ }^{3,4}$. There are three main complement pathways, each of which can distinguish self from nonself targets. The classical pathway is initiated by antibody recognition of a microbial target and subsequent binding of complement component $\mathrm{C} 1$ to the antigen-antibody complex. The lectin pathway recognizes specific patterns composed of saccharides and related molecules on microbial surfaces ${ }^{5,6}$. The alternative pathway, which is probably the oldest in evolutionary terms, does not involve a specific mechanism to distinguish non-self from self, relying instead on protection of self tissues using host cell surface molecules $^{7-9}$ (FIG. 1).

After their initial activation by different targets, all three pathways converge on cleavage of complement component $\underline{\mathrm{C}} 3$, which leads to the covalent attachment of fragment C3b to the target surface, and they end with the generation of the anaphylatoxin C5a and, in the case of Gram-negative bacteria, direct killing by the formation of pores in the target cell membranes (by formation of the membrane attack complex (MAC), composed of C5b, C6, C7, C8 and C9) (FIG. 1). In this context, the distinction between Grampositive bacteria (for example, staphylococci) and Gram-negative bacteria (for example, Neisseria meningitidis, also known as the meningococcus) becomes evident. Grampositive bacteria have a thick peptidoglycan layer that prevents incorporation of the MAC into the membrane, whereas Gramnegative bacteria are surrounded by two membranes, the inner and outer membranes, both of which can be attacked by the MAC; however, how MAC proteins reach the inner membrane is poorly understood. Fragment C3b tags bacteria for phagocytosis and subsequent killing by phagocytes and promotes antigen processing by antigen-presenting cells, thereby bridging the innate and adaptive immune responses. The generation of C5a is essential for attracting phagocytes to the site of infection and also for activating these cells for optimal phagocytosis. Thus, the removal of Gram-negative bacteria is mediated by phagocytes through $\mathrm{C} 3 \mathrm{~b}$ and
C5a and by direct killing by the complement system itself, whereas the removal of Grampositive bacteria is solely dependent on C3b, C5a and subsequent phagocytic killing .

Convertase molecules are crucial for all of these steps. On the surface of the bacterial target, $\mathrm{C} 3$ convertases are formed - either $\mathrm{C} 4 \mathrm{~b} 2 \mathrm{a}$ or $\mathrm{C} 3 \mathrm{bBb}$, the classical and alternative pathway convertases, respectively ( $\mathrm{C} 3 \mathrm{bBb}$ is discussed in detail below) - and these, in turn, deposit more and more $\mathrm{C} 3 \mathrm{~b}$ molecules on the surface of the target bacterium. These $\mathrm{C} 3$ convertases also bind additional C $3 \mathrm{~b}$ molecules to form $\mathrm{C} 5$ convertases. The $\mathrm{C} 5$ convertases cleave $\mathrm{C} 5$, resulting in the release of $\mathrm{C} 5 \mathrm{a}$, the sequential association of $\mathrm{C} 5 \mathrm{~b}$, $\mathrm{C} 6, \mathrm{C} 7, \mathrm{C} 8$ and $\mathrm{C} 9$, and the deposition of the assembled MAC.

To avoid destruction of host tissue, complement activation is inhibited on the surface of host cells, mainly at the level of $\mathrm{C} 3$, which is at the heart of the complement system. To mediate this inhibition, host cells express membrane-bound complement regulators such as complement decay-accelerating factor (DAF; also known as CD55), membrane cofactor protein (MCP; also known as CD46), complement receptor type 1 (CR1; also known as CD35) and complement receptor of the immunoglobulin superfamily (CRIg; also known as VSIG4), or markers (often polyanionic structures) that attract soluble complement regulators such as factor $\mathrm{H}$ (which downregulates activation of the alternative pathway) and the soluble negative regulator C4b-binding protein (C4BP) (which inhibits the classical and lectin pathways). Recent structural data have elucidated the molecular mechanisms that are responsible for the activation and regulation of $\mathrm{C} 3$ molecules.

Bacterial pathogens have evolved diverse strategies to avoid complement activation by mimicking host surfaces, attracting host complement regulators through surfaceexposed proteins, or targeting complement components with secreted proteins. The list of microbial molecules that have been reported to bind complement regulators is constantly growing (FIG. 1). Examples include: factor $\mathrm{H}$-binding protein ( $\mathrm{fHbp}$ ) from N. meningitidis ${ }^{10}$, outer surface protein E (OspE) and CspA from Borrelia burgdorferi $^{11,12}$, staphylococcal binder of 

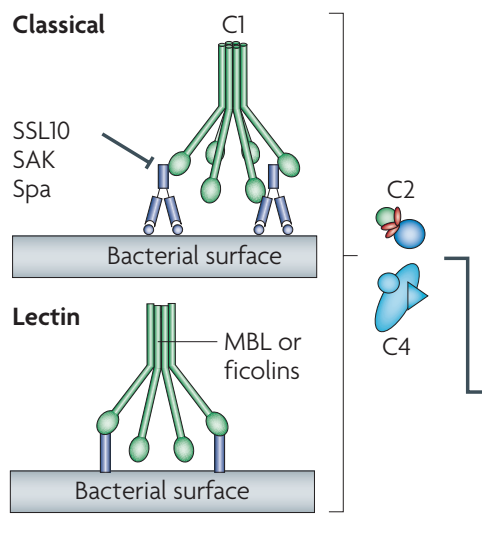

Alternative

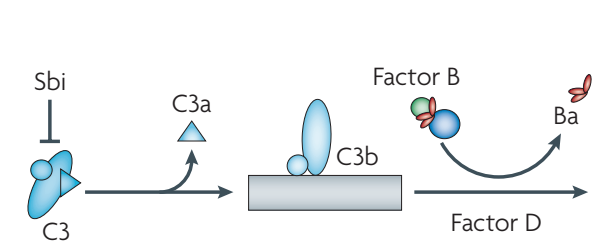

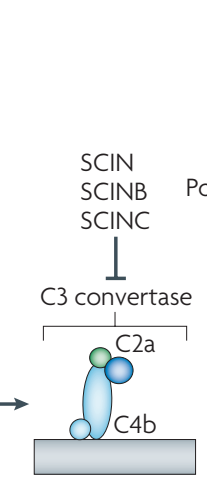
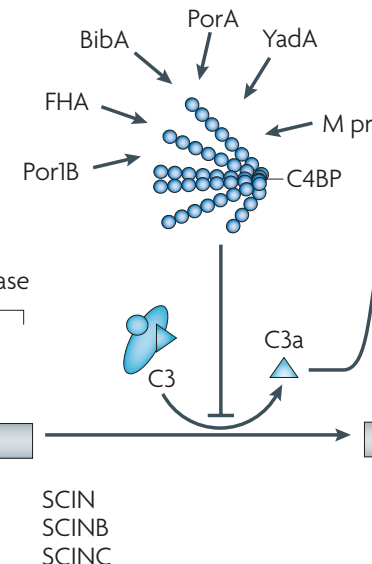

SCINC

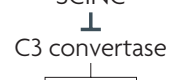

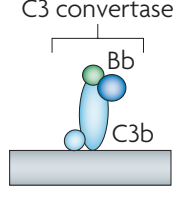

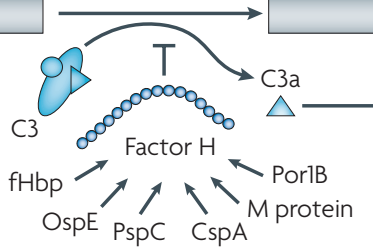

H-binding protein; FLIPr, formyl peptide receptor-like 1 inhibitory protein; MAC, membrane attack complex; MBL, mannose-binding lectin; OspE, outer surface protein E; PspC, pneumococcal surface protein C; SAK, staphylokinase; Sbi, staphylococcal binder of immunoglobulin; SCIN, staphylococcal complement inhibitor; Spa, staphylococcal protein A; SSL, staphylococcal superantigen-like; YadA, Yersinia adhesin $\mathrm{A}$.

immunoglobulins ( $\underline{\mathrm{Sbi}}$ ) from $\underline{\text { Staphylococcus }}$ aureus $^{13}$ and pneumococcal surface protein C (PspC) from Streptococcus pneumoniae ${ }^{14}$, which all bind factor $\mathrm{H}$; BibA from group B Streptococcus (also known as Streptococcus agalactiae $)^{15}$, filamentous haemagglutinin (FHA) from Bordetella pertussis ${ }^{16}$, PorA from N. meningitidis ${ }^{17}$ and Yersinia adhesin A (YadA) from Yersinia enterocolitica ${ }^{18}$, which all bind C4BP; and $\mathrm{M}$ protein from group A Streptococcus (also known as Streptococcus pyogenes), which binds both factor $\mathrm{H}$ and $\mathrm{C} \mathrm{BP}^{19,20}$. Additionally, clumping factor A (ClfA) of S. aureus binds to factor ${ }^{21}$. Once a given pathogen has recruited complement regulators to its surface, it is recognized as self and is protected from complement activation and amplification through the alternative pathway. In addition to expressing cell surface inhibitors, bacteria also use secreted molecules to inhibit complement deposition on their surface. S. aureus, for example, secretes several proteins that target different steps of the complement pathway. Both strategies allow bacteria to survive and successfully multiply in the host.
This Progress article takes advantage of the recent determination of the threedimensional structures of several complement components and their complexes to discuss advances in our understanding of the molecular mechanisms used by bacteria to evade complement activation. To aid our understanding of complement evasion at a molecular level, we first discuss recent insights into the molecular details of the amplification of complement activation by the alternative pathway and into complement regulation by the so-called regulators of complement activation (RCA) family. Next, we discuss the diverse evasion mechanisms deployed by bacterial pathogens, using as examples two human bacterial pathogens, S. aureus and N. meningitidis, which avoid complement mainly by using secreted and surface-exposed proteins, respectively.

\section{Insights into activation and regulation}

In recent years, detailed insights into complement activation and regulation have been provided by the determination of the structures of several key complement proteins $^{22,23}$ and, more recently, protein complexes involved in the alternative pathway. Moreover, structures of immune evasion molecules in complex with their target complement proteins have revealed how pathogens thwart the cascade of molecular events that normally form the complement defence system. Here, we consider the recent structural data obtained about complement amplification by $\mathrm{C} 3$ and factor $\mathrm{B}$ and about complement regulation by factor $\mathrm{H}$, which downregulates this amplification.

The structure of C3, the central molecule of the complement cascade, shows that this large, $187 \mathrm{kDa}$ protein is composed of 13 domains and that the reactive thioester is buried inside the core and thus protected from interaction with the environment ${ }^{24,25}$ (FIG. 2). Proteolysis of C3 removes the anaphylatoxin domain (the C3a fragment) to generate $\mathrm{C} 3 \mathrm{~b}^{26}$. The structures of $\mathrm{C} 3$ and $\mathrm{C} 3 \mathrm{~b}$ indicate that there are marked conformational changes on activation of native C3 into the reactive $\mathrm{C} 3 \mathrm{~b}$ species ${ }^{27,28}$. On removal of the C3a fragment, the remaining twelve domains of $\mathrm{C} 3 \mathrm{~b}$ rearrange to relocate the 


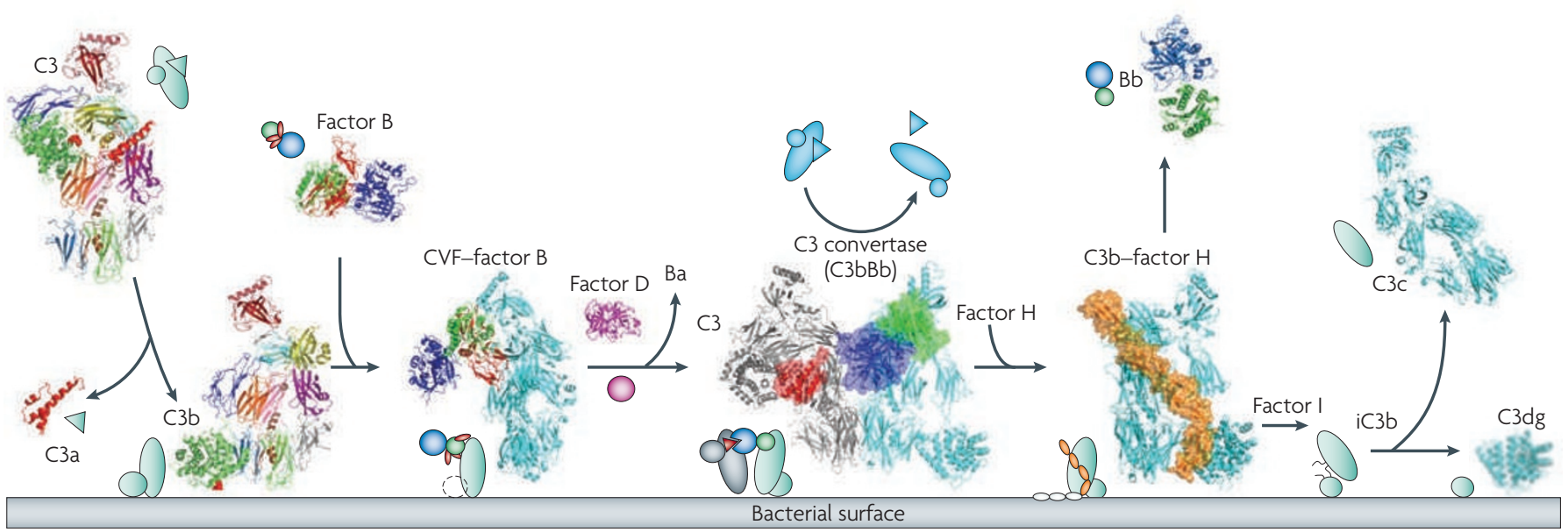

Figure 2 | The available structural data for the alternative pathway. The central molecule $\mathrm{C} 3$ consists of 13 domains, shown by the different colours in the left-most structure. C 3 is cleaved by the $\mathrm{C} 3$ convertase (generated either through the classical and lectin pathways or through the alternative pathway). Cleavage results in release of the small anaphylatoxin C3a and the large opsonin C3b. The activated C $3 b$ exposes its thioester and covalently binds to hydroxyls on adjacent surfaces. $\mathrm{C} 3 \mathrm{~b}$ binds pro-enzyme factor $\mathrm{B}$, which consists of five domains: the Von Willebrand factor A-type domain (green), the serine protease domain (dark blue) and the domains comprising fragment $\mathrm{Ba}$ (red). Binding of factor $\mathrm{B}$ to $\mathrm{C} 3 \mathrm{~b}$ is indicated by the complex formed by factor $\mathrm{B}$ and the $\mathrm{C} 3 \mathrm{~b}$ homologue cobra venom factor (CVF). Next,

factor $B$ is cleaved by factor $D$, yielding $C 3 b B b$, which is the active $C 3$ convertase of the alternative pathway. In the scheme, the putative binding of $\mathrm{C} 3$ to $\mathrm{C} 3 \mathrm{bBb}$ is indicated (here, $\mathrm{C} 3$ is in grey, with the $\mathrm{C} 3 \mathrm{a}$ domain in red; $\mathrm{C} 3 \mathrm{~b}$ is shown in light blue); the factor $\mathrm{B}$ protease fragment $\mathrm{Bb}$ (dark blue and green) is associated with the carboxyl terminus of the $\mathrm{C} 3$ substrate. The $\mathrm{C} 3$ convertase cleaves additional $\mathrm{C} 3$ molecules into $\mathrm{C} 3 \mathrm{a}$ and $\mathrm{C} 3 \mathrm{~b}$, thereby amplifying complement activation. Host cells are protected by complement regulators such as factor $\mathrm{H}$. Factor $\mathrm{H}$ dissociates the $\mathrm{C} 3 \mathrm{bBb}$ complex by binding to $\mathrm{C} 3 \mathrm{~b}$ (shown is the complex of $\mathrm{C} 3 \mathrm{~b}$ and factor $\mathrm{H}$ domains CCP1-CCP4). Subsequent binding of protease factor I cleaves $\mathrm{C} 3 \mathrm{~b}$ into inactive $\mathrm{C} 3 \mathrm{~b}(\mathrm{iC} 3 \mathrm{~b})$ and the further degradation products $\mathrm{C} 3 \mathrm{dg}$ and $\mathrm{C} 3 \mathrm{c}$.

thioester-containing domain (TED) and expose and chemically modify the reactive thioester moiety for covalent attachment to hydroxyl groups on the target surface ${ }^{26-28}$ (FIG. 2). The proteolysis of C3 is achieved by short-lived protease complexes, called C3 convertases, that form on target surfaces ${ }^{29,30}$. The classical and lectin pathways involve a C3 convertase that is formed by C4b and C2 (yielding a C4b2a complex), whereas the alternative pathway involves a homologous complex that is formed by $\mathrm{C} 3 \mathrm{~b}$ and factor $\mathrm{B}$. The C3 convertase of the alternative pathway yields a positive feedback loop that results in the amplification of $\mathrm{C} 3 \mathrm{~b}$ production and the deposition of many C3b molecules on target surfaces, causing opsonization ${ }^{24}$.

Structural data are now available for: the isolated inactive protease fragment of factor $\mathrm{B}$, known as the $\mathrm{Bb}$ fragment ${ }^{31}$ (and its $\mathrm{C} 2 \mathrm{a}$ homologue ${ }^{32,33}$ ); the native pro-enzyme form of factor $\mathrm{B}^{34}$; native $\mathrm{C} 3$ and its proteolytic fragments C3a, C3b, C3c and C3d 25,27,28,35,36; the pro-convertase complex formed by $\mathrm{C} 3 \mathrm{~b}$ and factor B (both low-resolution data from negative-stained electron microscopy ${ }^{37,38}$ and high-resolution data from X-ray diffraction of the $\mathrm{C} 3 \mathrm{~b}$ homologue cobra venom factor ${ }^{39}$ ); the protease factor $\mathrm{D}^{40}$, which is required to proteolytically activate $\mathrm{C} 3 \mathrm{bB}$ into $\mathrm{C} 3 \mathrm{bBb}$; and the convertase complex $\mathrm{C} 3 \mathrm{bBb}^{41}$ (FIG. 2). Briefly, the pro-enzyme factor B is first loaded onto $\mathrm{C} 3 \mathrm{~b}$, making contact through both the pro-peptide segment $\mathrm{Ba}$ and the protease segment $\mathrm{Bb}$. Factor B then changes from a 'loading' state to an 'activation' state, which probably exposes the scissile loop for cleavage by factor D. Cleavage by factor $\mathrm{D}$ removes the Ba segment (formed by the three amino-terminal domains and a linker), which results in the formation of the active, short-lived C3 convertase consisting of $\mathrm{C} 3 \mathrm{~b}$ and the two-domain protease, fragment $\mathrm{Bb}$. In this $\mathrm{C} 3 \mathrm{bBb}$ complex, the protease fragment is bound to the carboxyl terminus of $\mathrm{C} 3 \mathrm{~b}$ through a critical $\mathrm{Mg}^{2+}-$ dependent adhesion site that is formed by a Von Willebrand factor A-type domain. The serine protease domain with the catalytic centre is covalently attached to this domain and is oriented outwards.

Determination of the structure of the short-lived $\mathrm{C} 3 \mathrm{bBb}$ complex was facilitated by the use of staphylococcal complement inhibitor (SCIN) from S. aureus ${ }^{41}$. This secreted protein stabilizes and inhibits the convertase $\mathrm{e}^{42,43}$. The structure of the $\mathrm{C} 3 \mathrm{bBb}-\mathrm{SCIN}$ complex provided insights into both the activity and specificity of the convertase, as well as the inhibitory mechanism of SCIN (FIG. 3a). The observed dimeric $\mathrm{C} 3 \mathrm{bBb}-\mathrm{C} 3 \mathrm{bBb}$ complex, which is stabilized by SCIN, suggests that the substrate specificity of the convertase is determined by $\mathrm{C} 3$ binding to $\mathrm{C} 3 \mathrm{~b}$ and forming a $\mathrm{C} 3-\mathrm{C} 3 \mathrm{bBb}$ complex, with $\mathrm{C} 3-\mathrm{C} 3 \mathrm{~b}$ in a heterodimeric arrangement. Positioning C3 in place of one of the $\mathrm{C} 3 \mathrm{~b}$ molecules brings the scissile bond of $\mathrm{C} 3$ in front of the catalytic site of the serine protease domain of the opposing $\mathrm{C} 3 \mathrm{bBb}$ complex. Three inhibitors that block substrate binding to the convertase are known. The structures of these inhibitors bound to $\mathrm{C} 3 \mathrm{~b}$ (or the breakdown product C3c) ${ }^{28,44,45}$ show, indeed, that they sterically block the formation of the $\mathrm{C} 3-\mathrm{C} 3 \mathrm{bBb}$ complex. Obviously, SCIN-mediated stabilization of the $\mathrm{C} 3 \mathrm{bBb}-\mathrm{C} 3 \mathrm{bBb}$ complex also results in the blocking of $\mathrm{C} 3$ substrate binding and, thus, in inhibition of the convertase. However, a chimeric variant of SCIN inhibited convertase activity but did not induce dimerization. In this case, SCIN probably obstructs the ability of the serine protease domain to bind the scissile loop of the substrate.

Structural information has also contributed to our understanding of the regulation of complement activity. Proteins of the RCA family consist of strings of complement control protein (CCP) domains and function through two possible mechanisms. Most regulators, such as MCP, DAF and CR1, are surface bound. By contrast, factor $\mathrm{H}$ is an abundant soluble regulator that consists of 20 CCP domains. Recognition of host cells and surfaces by factor $\mathrm{H}$ can be attributed to the binding of polyanions such as glycosaminoglycans by domains 
a

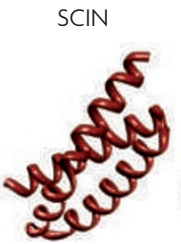

b
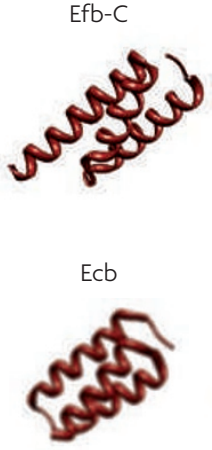

d
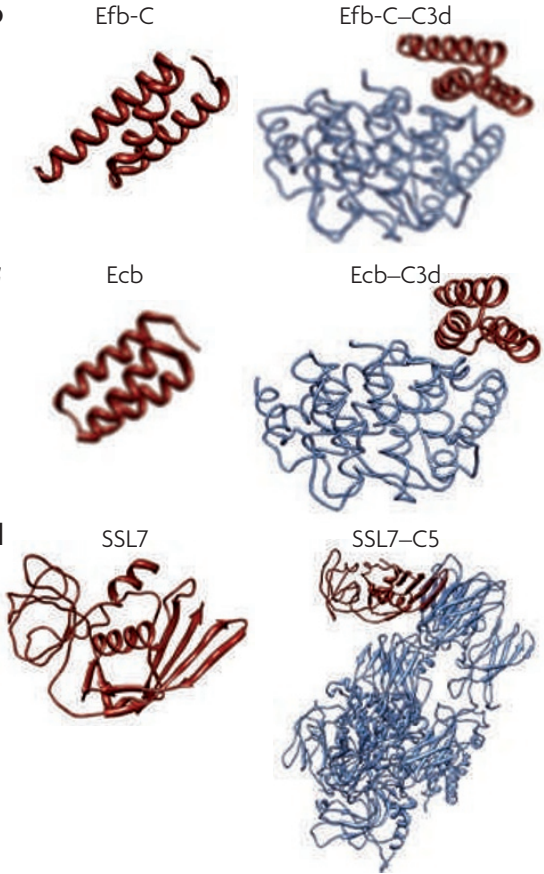

e
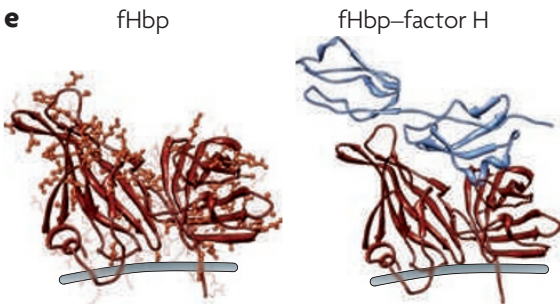

Figure $3 \mid$ Structures of bacterial proteins that bind complement components and of their complexes with complement proteins. a Staphylococcal complement inhibitor (SCIN) (Protein Data Bank (PDB) entry 2QFF) and its dimeric complex with the $\mathrm{C} 3$ convertase of the alternative pathway, C3bBb (PDB entry 2WIN). b | The staphylococcal extracellular fibrinogenbinding protein (Efb; also known as Fib) carboxyterminal domain (PDB entry $2 \mathrm{GOM}$ ), and Efb-C in complex with C3d (PDB entry 2GOX). c | The staphylococcal extracellular complement-binding protein (Ecb; also known as Ehp) and the complex with C3d (PDB entry 2NOJ). d | Staphylococcal superantigen-like protein 7 (SSL7) and the complex with C5 (PDB entry 3KLS). e | Factor $\mathrm{H}$-binding protein ( $\mathrm{fHbp}$ ) of Neisseria meningitidis (PDB entry $2 \mathrm{KCO}$ ) and its complex with factor H (PDB entry $2 \mathrm{~W} 80$ ). Complement proteins are in blue, and bacterial proteins are in red. Variable residues of $\mathrm{fHbp}$ are rendered as ball-and-stick drawings.
CCP6-CCP8 and by the C-terminal domains CCP19-CCP20 (these C-terminal domains form a ternary complex with both surface glycans and the TED domain of C $3 b)^{46,47}$. Domains CCP1-CCP4 harbour the functional activity of factor $\mathrm{H}^{48}$. The structure of $\mathrm{C} 3 \mathrm{~b}$ in complex with CCP1-CCP4 of factor $\mathrm{H}$ shows that the CCP domains bind in an extended fashion to $\mathrm{C}^{3} \mathrm{~b}^{49}$ (FIG. 2). This arrangement indicates that domains CCP1-CCP2 are directly responsible for dissociating the $\mathrm{C} 3 \mathrm{bBb}$ complex, giving rise to the so-called decay-accelerating activity and stopping $\mathrm{C} 3 \mathrm{~b}$ production. As an alternative mechanism, $\mathrm{C} 3 \mathrm{~b}$-factor $\mathrm{H}$ binds the protease factor I, which cleaves the CUB domain of C3b. A putative binding site for factor I is formed by the C-terminal domain of $\mathrm{C} 3 \mathrm{~b}$ and domains $\mathrm{CCP} 2-\mathrm{CCP} 3$ of factor $\mathrm{H}$, which lie adjacent to the CUB domain in the $\mathrm{C} 3 \mathrm{~b}-$ factor $\mathrm{H}$ complex. Cleavage of C3b by factor I results in $\mathrm{iC} 3 \mathrm{~b}$, which does not support formation of the convertase complex and thereby stops the local production of $\mathrm{C} 3 \mathrm{~b}$. These host protection molecules have been adopted by vaccinia and variola viruses, which express proteins consisting of four CCP domains on their surface, mimicking the mechanisms of host factor $\mathrm{H}$. Bacterial pathogens have also evolved proteins that bind the soluble regulators, as is the case for meningococcal fHbp.

\section{Soluble complement inhibitors}

S. aureus is a major pathogen that is responsible for both nosocomial and communityacquired infections and that has developed sophisticated mechanisms to adapt, persist and cause infection in different host environments ${ }^{50}$. Numerous secreted proteins that block the cellular receptors and serum proteins involved in innate immunity have been identified and characterized in S. aureus and, interestingly, many of these are complement inhibitors.

Staphylococcal protein A (Spa), staphylococcal binder of immunoglobulins (Sbi) and staphylococcal superantigen-like protein 10 ( $\underline{\text { SSL10) }}$ ) bind immunoglobulin G (IgG) that is deposited on the surface of the bacterium and interfere with activation of the classical pathway $^{50-52}$. Staphylokinase (SAK) removes IgG from the surface of bacteria indirectly, by cleaving $\mathrm{it}^{50}$. These proteins also influence IgG-mediated phagocytosis at a later stage of the immune response. Other proteins act at the next level of complement activation and inhibit the C3 convertases. SCIN and its homologues SCINB and SCINC bind and inhibit both the classical pathway convertase, $\mathrm{C} 4 \mathrm{~b} 2 \mathrm{a}$, and the alternative pathway convertase, $\mathrm{C} 3 \mathrm{bBb}^{41,42}$. As discussed above, SCIN blocks, stabilizes and dimerizes $\mathrm{C} 3 \mathrm{bBb}$ (a detail that was used to resolve the threedimensional structure of this convertase). The formation of $\mathrm{C} 3 \mathrm{bBb}$ dimers inhibits complement-mediated phagocytosis by blocking the receptor sites on $\mathrm{C}_{3} \mathrm{~b}^{53}$. In addition, other proteins from $S$. aureus act on C3 and its proteolytic fragments. Sbi inhibits complement activation by binding factor $\mathrm{H}$ when it is in combination with another complement protein $(\mathrm{C} 3, \mathrm{C} 3 \mathrm{~b}$ or $\mathrm{C} 3 \mathrm{~d})$ adjacent to binding immunoglobulins ${ }^{13}$. Extracellular fibrinogen-binding protein (Efb; also known as Fib) and the homologous extracellular complement-binding protein (Ecb; also known as Ehp) bind to the TED domain present in C3, C3b and C3 $d^{54,55}$ (FIG. 3b,c). Functionally, the binding of Efb and Ecb to the TED domain blocks the alternative pathway convertase and, thus, subsequent $\mathrm{C} 3 \mathrm{~b}$ deposition. Furthermore, these proteins down-modulate the function of both the alternative pathway and the classical pathway C5 convertases (presumably by binding the TED domain of the $\mathrm{C} 3 \mathrm{~b}$ molecule in these complexes), resulting in diminished C5a generation ${ }^{56}$. SSL7 binds to $\mathrm{C} 5$ and, through an alternative mechanism, inhibits the conversion of $\mathrm{C} 5$ into C $5 \mathrm{a}^{57}$. These examples show that bacterial pathogens use various complement-binding proteins to block the complement cascade at different stages. This was also shown by the recent observation that SCINB and Efb are produced simultaneously, as they are located in a single operon, and act synergistically to inhibit all C3 and C5 convertases ${ }^{58}$.

The anaphylatoxin C5a stimulates a strong inflammatory response. The expression of P-selectin in the local endothelium is increased under the influence of C5a, formylated peptides and other secondary chemokines, paving the way for neutrophil rolling, adherence and transmigration. In this way, neutrophils can extravasate and move up the gradient of chemoattractants to the site of infection. S. aureus has evolved an armamentarium of weapons for this phase of infection. SSL5 blocks neutrophil extravasation at two crucial points: first, it inhibits a crucial interaction between neutrophils and the endothelium (the P-selectin glycoprotein ligand 1-P-selectin interaction), and second, it blocks the activation of $\mathrm{G}$ protein-coupled receptors (GPCRs) by chemokines $^{59}$. Extracellular adherence protein (Eap) blocks the next step in neutrophil extravasation, blocking firm adhesion of neutrophils to the endothelium by interfering with the integrin-intercellular adhesion 
molecule 1 interaction ${ }^{60}$. S. aureus also interferes with the next steps in neutrophil movement: the chemotaxis, priming and activation events that are mediated mainly by GPCRs on the neutrophil surface. SSL10 blocks CXC-chemokine receptor 4 (CXCR4) and, thus, the action of CXC-chemokine ligand $12(\mathrm{CXCL} 12)^{61}$. Chemotaxis inhibitory protein of staphylococci ( $\underline{\mathrm{CHIPS}})^{62}$ inhibits C5a receptor (C5AR1) as well as formyl peptide receptor (FPR1), thereby blocking the activation of formylated peptides as well as the potent anaphylatoxin C5a. Thus, staphylococci have evolved SSL7 to bind the C5 molecule itself and prevent its cleavage ${ }^{63}$, can block both $\mathrm{C} 5$ convertases using Efb and $\mathrm{Ecb}^{56}$, and can also block the receptor for C5a using CHIPS. All of these factors are expressed simultaneously in most clinical isolates of staphylococci and provide a seemingly redundant but possibly synergistic inhibition. FPR-like 1 (FPRL1) inhibitory protein (FLIPr) inhibits FPRL1, a homologue of FPR1, and FLIPr-like protein inhibits the activation of neutrophils by both FPR1 and FPRL1 (also known as FPR2) ${ }^{64}$. Finally, $S$. aureus also secretes proteins that interact with the process of phagocytosis itself, such as SSL10, Spa and Sbi (which act on IgGmediated phagocytosis), SAK (which cleaves C3b and IgG) and SSL7 (which binds IgA and C5 simultaneously) $)^{50-52,57}$. The recently obtained crystal structure of the C5-SSL7 complex confirms that binding to $\mathrm{C} 5$ occurs exclusively through the $\mathrm{C}$-terminal $\beta$-grasp domain of SSL7, leaving the OB domain free to interact with $\operatorname{IgA}^{63}$ (FIG. 3d). Once inside the neutrophil, various molecules protect the bacterium against the attack of proteases and oxygen radicals, but that is beyond the scope of this article.

Although the majority of proteins that inhibit complement and neutrophils are secreted, some cell wall-anchored proteins on staphylococci also act in this way. Recently, ClfA was shown to inhibit phagocytosis by binding to factor $\mathrm{I}^{21,65}$; furthermore, adenosine synthethase inhibits general neutrophil function $^{66}$ and iron-regulated surface determinant protein $\mathrm{H}$ ( $\mathrm{IsdH}$ ) inhibits complement activation by enhancing C3b degradation ${ }^{67}$. Sbi and Spa are also present on the bacterial surface and can fulfil their functions at this location as well as functioning as soluble proteins $s^{50,51}$.

Thus, staphylococci have evolved an arsenal of molecules to counteract the complement system. Using a plethora of small secreted proteins is still a highly energyefficient way to evade the innate immune response compared with, for example, capsule formation.
Complement evasion by meningococci

N. meningitidis is a Gram-negative, capsulated bacterium that causes meningococcal meningitis and septicaemia. The bacterium is adapted to the human host and is carried asymptomatically in the nasopharynx of approximately $5-10 \%$ of the human population $^{68}$. N. meningitidis uses the surfaceexposed proteins PorA and $\mathrm{fHbp}$ to bind $\mathrm{C} \mathrm{BP}^{69}$ and human factor $\mathrm{H}^{70,71}$, respectively. The general mechanisms of N. meningitidis resistance to host immunity have been recently reviewed elsewhere ${ }^{72}$; here, we focus on fHbp-mediated regulation of the alternative pathway, as the three-dimensional structure of fHbp has now been solved.

$\mathrm{fHbp}$ is a surface-exposed lipoprotein that was first identified by genomic screening as a protein antigen able to induce bactericidal antibodies $^{73}$. It was initially termed genomederived Neisseria antigen 1870 (GNA1870). The same protein was also identified through a biochemical approach and named LP2086 (REF. 74). Later, GNA1870 was found to bind factor $\mathrm{H}$ and was renamed $\mathrm{fHbp}$. By recruiting factor $\mathrm{H}$ to its surface, $N$. meningitidis resembles self tissue and is no longer attacked by complement, so can survive and grow in the bloodstream ${ }^{71,75}$. When it was later found that fHbp binds only human factor $\mathrm{H}$ and not factor $\mathrm{H}$ from mouse, rat or lower primates, it became clear why N. meningitidis is a human-specific pathogen and why we have so far been unable to develop reliable animal models of $N$. meningitidis infection. Whereas fHbp allows survival of the bacterium in human blood, in animal blood it does not recruit factor $\mathrm{H}$ and so the bacterium is killed by complement ${ }^{76}$. This suggests that mice that are transgenic for human factor $\mathrm{H}$ might serve as good animal models for meningococcal disease. Finally, binding of factor $\mathrm{H}$ by fHbp might also explain, in part, the devastating haemorrhagic syndrome that is associated with meningococcal septicaemia: recruitment of factor $\mathrm{H}$ by bacteria that have grown to high density in the bloodstream could deplete factor $\mathrm{H}$ in the surrounding tissue, so this self tissue becomes prone to attack by complement.

The three-dimensional structure of $\mathrm{fHbp}$, which was solved by nuclear magnetic resonance (NMR), revealed that this protein consists of two domains that are both rich in $\beta$-strands and are arranged in a butterflylike structure ${ }^{77-79}$. A flexible N-terminal tail anchors the protein to the bacterial cell through a lipid chain that is covalently attached to the first cysteine residue. The sequence variability to which $\mathrm{fHbp}$ is prone among the meningococcal population is concentrated mostly on one side of the molecule. Such a distribution indirectly suggests the orientation of fHbp with respect to the bacterial outer membrane, with the groove that separates the $\mathrm{N}$ - and $\mathrm{C}$-terminal domains oriented towards the external environment (FIG. 3e).

This orientation is also favourable for the interaction with domains CCP6 and CCP7 of human factor $\mathrm{H}$, as depicted by the recentlypublished crystal structure of this complex ${ }^{80}$ (FIG. 3e). Although the interaction between CCP7 and fHbp is limited and ascribed to just two loops of the N-terminal domain of the bacterial protein, CCP6 accommodates itself on the fHbp groove owing to good shape complementarity and a strong electrostatic interaction between His371 and Lys351 of CCP6 and Glu218 and Glu239 of $\mathrm{fHbp}$, respectively. These two residue pairs determine the efficacy of the interaction. Glu218Ala and Glu239Ala mutations dramatically reduce human factor $\mathrm{H}$ binding. Further, CCP6 is in the region of factor $\mathrm{H}$ that is responsible for the recognition of host cell polyanions. This suggests that meningococci evolved the ability to recruit human factor $\mathrm{H}$ to their surface using a surfaceexposed protein that mimics the function of polyanions in host carbohydrates. The crystal structure of the fHbp-factor $\mathrm{H}$ complex also reveals that the surface area of factor $\mathrm{H}$ that interacts with $\mathrm{fHbp}$ consists of residues that are not conserved between humans and lower vertebrates. This provides a structural rationale for the strict human specificity of fHbp and the bacterium itself ${ }^{76}$. Taken together, this suggests that binding of fHbp to factor H domains CCP6 and CCP7 localizes factor $\mathrm{H}$ to the meningococcal surface, where it then provides protection against complement activation by binding to deposited C3b molecules (through the factor $\mathrm{H}$ domains CCP1-CCP4) and, thus, blocks amplification of the complement response.

The presence of a factor $\mathrm{H}$-binding protein on the meningococcal surface outlines the relevance of an escape mechanism to avoid the killing mediated by the alternative pathway of the complement system. However, it was recently suggested that the alternative pathway acts mainly by amplifying the complement activation that is initially triggered by the classical pathway ${ }^{81}$.

\section{Implications for vaccine development} Surface-exposed molecules that can be targets for complement-activating antibodies are known to be excellent vaccine antigens. Examples include the capsular polysaccharides of pneumococcus and meningococcus 
serogroups A, C, W135 and Y, for which the polysaccharides, delivered alone or as protein-polysaccharide conjugates, are part of effective licensed vaccines. More recently, genome-wide screening (also known as reverse vaccinology) has identified several novel vaccine candidate proteins that are good targets for complement-fixing antibodies. The most notable examples are $\mathrm{fHbp}$, Neisseria adhesin A (NadA) and neisserial heparin-binding antigen (NHBA; also known as GNA2132) from N. meningitidis, which are now in phase III clinical trials, and pili from group A Streptococcus, group B Streptococcus and S. pneumoniae. In some cases, as well as being targets for complement-fixing antibodies, some antigens can interact directly with complement components. This is the case for fHbp of N. meningitidis, BibA of group B Streptococcus, PspC of $S$. pneumoniae and FHA of B. pertussis ${ }^{82}$.

fHbp can be considered a unique $N$. meningitidis vaccine antigen. Antibodies directed against fHbp have several modes of action. Those with serum bactericidal activity can mediate direct bacteriolysis through the classical complement pathway and also promote phagocytosis and subsequent intracellular killing. In parallel, fHbp-specific antibodies can block binding of factor $\mathrm{H}$, increasing bacterial susceptibility to killing by the alternative pathway ${ }^{71,75,83}$. In addition, as complement activation would no longer be inhibited, more C5a would be generated, and phagocyte activation and subsequent phagocytosis and killing would be promoted. This suggests that using specific antibodies to block the activity of a complement inhibitor could be a potent strategy to design new vaccines for a large variety of bacteria. This is not a new strategy, if we consider the fact that the oldest known complement evasion strategy is the capsule, and we have been making effective capsule vaccines for decades. By understanding why antigens that bind to human complement components are good vaccine antigens, it might be possible to define the molecular basis of an ideal vaccine antigen. Understanding the molecular nature and the functional consequences of targeting certain candidate antigens will be instrumental in providing a scientific basis to guide the selection of vaccine targets and vaccine design. Furthermore, determining the three-dimensional structures of the complexes formed by pathogenic proteins and their complement targets will be advantageous for our understanding of the molecular mechanisms of complement evasion, as highlighted by SCIN, Efb, SSL7 and fHbp.
Although many factor $\mathrm{H}$ - and C4BPbinding proteins on the surface of various bacteria have been described, recent work on staphylococci indicates that most innate immune evasion molecules in these species are small secreted proteins. In part, this may reflect the difference between the surfaces of Gram-negative and Gram-positive species. In Gram-negative bacteria, membrane proteins or membrane-anchored proteins are free to move and can therefore cover the whole bacterial surface to prevent complement activation. On the surface of Grampositive bacteria, however, cell wall-anchored proteins cannot reach and cover every part of the bacterial surface unless the density of the protein is extremely high. As indicated for staphylococci, secreted molecules can surround the bacterial environment and act on complement deposition on the surface by inhibiting various complement components at diverse stages of complement activation. So, for several aspects of immune attack, secreted molecules are an effective method of counterattack by Gram-positive species. However, this does not rule out a function for some cell surface-bound molecules in immune evasion. Furthermore, Gram-positive organisms are insensitive to complement-mediated lysis, and removal of these pathogens is dependent only on phagocytes. Therefore, in developing evasion strategies, these pathogens have also evolved factors that specifically target complement receptors or other structures on phagocytes, and secreted proteins are again more suitable for such strategies than surface-associated proteins.

The complement system plays a crucial role in the host defence against systemic disease caused by N. meningitidis. This is proved by the fact that deficiencies of the complement system that affect the alternative pathway and the terminal complement components such as the MAC have been associated with increased susceptibility to meningococcal infections ${ }^{84}$. However, the same host deficiencies have not been associated with increased susceptibility to staphylococcal infections. This suggests that the complement system is the main innate host defence mechanism against $N$. meningitidis, but that a combination of complement and cellular innate immunity defends the host against $S$. aureus. This is in agreement with the observation that neutropenic patients are highly susceptible to staphyloccocal infection.

In conclusion, focusing on the microbial molecules that interact with human complement has revealed important information about microbial pathogenesis. Elucidating the molecular mechanisms underlying complement evasion will help to develop novel vaccine antigens and, in the meantime, improve our understanding of the molecular and functional basis of good vaccine antigens.

Davide Serruto, Rino Rappuoli and Maria Scarselli are at Novartis Vaccines and Diagnostics, Via Fiorentina 1, 53100 Siena, Italy.

Piet Gros is at Crystal and Structural Chemistry, Bijvoet Center for Biomolecular Research, Department of Chemistry, Faculty of Science, Utrecht University, Padualaan 8, $3584 \mathrm{CH}$ Utrecht, The Netherlands.

Jos A. G. van Strijp is at the Department of Medical Microbiology, University Medical Center Utrecht, Heidelberglaan 100, 3584 CX, Utrecht, The Netherlands.

Correspondence to R.R. e-mail:rino.rappuoli@novartis.com doi: 10.1038/nrmicro2366

. Jongerius, I., Ram, S. \& Rooijakkers, S. Bacterial complement escape. Adv. Exp. Med. Biol. 666, 32-48 (2009).

2. Lambris, J. D., Ricklin, D. \& Geisbrecht, B. V. Complement evasion by human pathogens. Nature Rev. Microbiol. 6, 132-142 (2008).

3. Walport, M. J. Complement. First of two parts. N. Engl. J. Med. 344, 1058-1066 (2001).

4. Walport, M. J. Complement. Second of two parts. N. Engl. J. Med. 344, 1140-1144 (2001).

5. Thiel, S. Complement activating soluble pattern recognition molecules with collagen-like regions, mannan-binding lectin, ficolins and associated proteins. Mol. Immunol. 44, 3875-3888 (2007).

6. Krarup, A., Mitchell, D. A. \& Sim, R. B. Recognition of acetylated oligosaccharides by human L-ficolin. Immunol. Lett. 118, 152-156 (2008).

Joiner, K. A. Complement evasion by bacteria and parasites. Annu. Rev. Microbiol. 42, 201-230 (1988)

8. Meri, S. \& Pangburn, M. K. Discrimination between activators and nonactivators of the alternative pathway of complement: regulation via a sialic acid/ polyanion binding site on factor H. Proc. Natl Acad. Sci. USA 87, 3982-3986 (1990).

9. Pangburn, M. K. \& Muller-Eberhard, H. J. The alternative pathway of complement. Semin. Immunopathol. 7, 163-192 (1984).

10. Pizza, M., Donnelly, J. \& Rappuoli, R. Factor H-binding protein, a unique meningococcal vaccine antigen. Vaccine 26 (Suppl. 8), 146-148 (2008).

11. Hellwage, J. et al. The complement regulator factor $\mathrm{H}$ binds to the surface protein OspE of Borrelia burgdorferi. J. Biol. Chem. 276, 8427-8435 (2001).

12. Kraiczy, P. et al. Complement resistance of Borrelia burgdorferi correlates with the expression of BbCRASP-1, a novel linear plasmid-encoded surface protein that interacts with human factor $\mathrm{H}$ and FHL-1 and is unrelated to Erp proteins. J. Biol. Chem. 279, 2421-2429 (2004).

13. Haupt, K. et al. The Staphylococcus aureus protein Sbi acts as a complement inhibitor and forms a tripartite complex with host complement Factor $\mathrm{H}$ and C3b. PLoS Pathog. 4, e 1000250 (2008)

14. Janulczyk, R., lannelli, F., Sjoholm, A. G., Pozzi, G. \& Bjorck, L. Hic, a novel surface protein of Streptococcus pneumoniae that interferes with complement function. J. Biol. Chem. 275, 37257-37263 (2000).

15. Santi, l. et al. BibA: a novel immunogenic bacterial adhesin contributing to group B Streptococcus survival in human blood. Mol. Microbiol. 63 754-767 (2007).

16. Merianos, A. Surveillance and response to disease emergence. Curr. Top. Microbiol. Immunol. 315 477-509 (2007).

17. Jarva, H. et al. The group B streptococcal $\beta$ and pneumococcal Hic proteins are structurally related immune evasion molecules that bind the complement inhibitor factor $\mathrm{H}$ in an analogous fashion. J. Immunol. 172, 3111-3118 (2004).

18. Kirjavainen, V. et al. Yersinia enterocolitica serum resistance proteins YadA and Ail bind the complement regulator C4b-binding protein. PLoS Pathog. 4 e1000140 (2008). 
19. Horstmann, R. D., Sievertsen, H. J., Knobloch, J. \& Fischetti, V. A. Antiphagocytic activity of streptococcal $\mathrm{M}$ protein: selective binding of complement control protein factor H. Proc. Natl Acad. Sci. USA 85 , 1657-1661 (1988).

20. Johnsson, E. et al. A highly variable region in members of the streptococcal $\mathrm{M}$ protein family binds the human complement regulator C4BP. J. Immunol. 157, 3021-3029 (1996).

21. Hair, P. S., Ward, M. D., Semmes, O. J., Foster, T. J. \& Cunnion, K. M. Staphylococcus aureus clumping factor A binds to complement regulator factor I and increases factor I cleavage of C3b. J. Infect. Dis. 198, 125-133 (2008)

22. Arlaud, G. J., Barlow, P. N., Gaboriaud, C., Gros, P. \& Narayana, S. V. Deciphering complement mechanisms: the contributions of structural biology. Mol. Immunol. 44, 3809-3822 (2007)

23. Gros, P., Milder, F. J. \& Janssen, B. J. C. Complement driven by conformational changes. Nature Rev. Immunol. 8, 48-58 (2008).

24. Law, S. K. \& Levine, R. P. Interaction between the third complement protein and cell surface macromolecules. Proc. Natl Acad. Sci. USA 74, 2701-2705 (1977).

25. Janssen, B. J. et al. Structures of complement component $\mathrm{C} 3$ provide insights into the function and evolution of immunity. Nature 437, 505-511 (2005).

26. Law, S. K. \& Dodds, A. W. The internal thioester and the covalent binding properties of the complement proteins C3 and C4. Protein Sci. 6, 263-274 (1997).

27. Janssen, B. J., Christodoulidou, A., McCarthy, A. Lambris, J. D. \& Gros, P. Structure of C3b reveals conformational changes that underlie complement activity. Nature 444, 213-216 (2006).

28. Wiesmann, C. et al. Structure of C3b in complex with CRIg gives insights into regulation of complement activation. Nature 444, 217-220 (2006).

29. Muller-Eberhard, H. J., Polley, M. J. \& Calcott, M. A. Formation and functional significance of a molecular complex derived from the second and the fourth component of human complement. J. Exp. Med. 125 359-380 (1967).

30. Muller-Eberhard, H. J. \& Gotze, O. C3 proactivator convertase and its mode of action. J. Exp. Med. 135 1003-1008 (1972).

31. Ponnuraj, K. et al. Structural analysis of engineered $\mathrm{Bb}$ fragment of complement factor $\mathrm{B}$ : insights into the activation mechanism of the alternative pathway C3-convertase. Mol. Cell 14, 17-28 (2004).

32. Milder, F. J. et al. Structure of complement component C2a: implications for convertase formation and substrate binding. Structure 14, 1587-1597 (2006).

33. Krishnan, V., Xu, Y., Macon, K., Volanakis, J. E. \& Narayana, S. V. L. The crystal structure of C2a, the catalytic fragment of classical pathway $\mathrm{C} 3$ and $\mathrm{C} 5$ convertase of human complement. J. Mol. Biol. 367 , 224-233 (2007).

34. Milder, F. J. et al. Factor B structure provides insights into activation of the central protease of the complement system. Nature Struct. Mol. Biol. 14 224-228 (2007)

35. Huber, R., Scholze, H., Paques, E. P. \& Deisenhofer, J. Crystal structure analysis and molecular model of human C3a anaphylatoxin. Hoppe Seylers Z. Physiol. Chem. 361, 1389-1399 (1980).

36. Nagar, B., Jones, R. G., Diefenbach, R. J., Isenman, D. E. $\&$ Rini, J. M. X-ray crystal structure of C3d: a C3 fragment and ligand for complement receptor 2 . Science 280, 1277-1281 (1998).

37. Torreira, E., Tortajada, A., Montes, T., Rodriguez de Cordoba, S. \& Llorca, O. 3D structure of the C3bB complex provides insights into the activation and regulation of the complement alternative pathway convertase. Proc. Natl Acad. Sci. USA 106, 882-887 (2009).

38. Torreira, E., Tortajada, A., Montes, T., Rodriguez de Cordoba, S. \& Llorca, O. Coexistence of closed and open conformations of complement factor $\mathrm{B}$ in the alternative pathway $\mathrm{C} 3 \mathrm{bB}\left(\mathrm{Mg}^{2+}\right)$ proconvertase. J. Immunol. 183, 7347-7351 (2009).

39. Janssen, B. J. et al. Insights into complement convertase formation based on the structure of the factor B-cobra venom factor complex. EMBO J. 28 2469-2478 (2009).

40. Narayana, S. V. et al. Structure of human factor D. A complement system protein at 2.0 A resolution. J. Mol. Biol. 235, 695-708 (1994)

41. Rooijakkers, S. H. et al. Structural and functional implications of the alternative complement pathway C3 convertase stabilized by a staphylococcal inhibitor. Nature Immunol. 10, 721-727 (2009).
42. Rooijakkers, S. H. M. et al. Immune evasion by a staphylococcal complement inhibitor that acts on C3 convertases. Nature Immunol. 6, 920-927 (2005).

43. Rooijakkers, S. H. M. et al. Staphylococcal complement inhibitor: structure and active sites. J. Immunol. 179, 2989-2998 (2007).

44. Katschke, K. J. Jr et al. Structural and functional analysis of a C3b-specific antibody that selectively inhibits the alternative pathway of complement. J. Biol. Chem. 284, 10473-10479 (2009).

45. Janssen, B. J., Halff, E. F., Lambris, J. D. \& Gros, P. Structure of compstatin in complex with complement component $\mathrm{C} 3 \mathrm{c}$ reveals a new mechanism of complement inhibition. J. Biol. Chem. 282 29241-29247 (2007).

46. Schmidt, C. Q. et al. A new map of glycosaminoglycan and $\mathrm{C} 3 \mathrm{~b}$ binding sites on factor, H. J. Immunol. 181, 2610-2619 (2008)

47. Prosser, B. E. et al. Structural basis for complement factor $\mathrm{H}$ linked age-related macular degeneration. J. Exp. Med. 204, 2277-2283 (2007)

48. Gordon, D. L., Kaufman, R. M., Blackmore, T. K., Kwong, J. \& Lublin, D. M. Identification of complement regulatory domains in human factor $\mathrm{H}$. J. Immunol. 155, 348-356 (1995).

49. Wu, J. et al. Structure of complement fragment C3bfactor $\mathrm{H}$ and implications for host protection by complement regulators. Nature Immunol. 10 728-733 (2009)

50. Rooijakkers, S. H., van Kessel, K. P. \& van Strijp, J. A Staphylococcal innate immune evasion. Trends Microbiol. 13, 596-601 (2005)

51. Burman, J. D. et al. Interaction of human complement with Sbi, a staphylococcal immunoglobulin-binding protein: indications of a novel mechanism of complement evasion by Staphylococcus aureus. J. Biol. Chem. 283, 17579-17593 (2008)

52. Itoh, S. et al. Staphylococcal superantigen-like protein 10 (SSL10) binds to human immunoglobulin G (IgG) and inhibits complement activation via the classical pathway. Mol. Immunol. 47, 932-938 (2010).

53. Jongerius, I. et al. Staphylococcal complement inhibitor modulates phagocyte responses by dimerization of convertases. J. Immunol. 184, 420-425 (2010).

54. Hammel, M. et al. A structural basis for complement inhibition by Staphylococcus aureus. Nature Immunol. 8, 430-437 (2007)

55. Hammel, M. et al. Characterization of Ehp, a secreted complement inhibitory protein from Staphylococcus aureus. J. Biol. Chem. 282, 30051-30061 (2007).

56. Jongerius, I. et al. Staphylococcal complement evasion by various convertase-blocking molecules. J. Exp. Med. 204, 2461-2471 (2007)

57. Langley, R. et al. The staphylococcal superantigen-like protein 7 binds IgA and complement C5 and inhibits IgA-FcaRI binding and serum killing of bacteria. J. Immunol. 174, 2926-2933 (2005)

58. Ten Broeke-Smits, N. J. et al. Operon structure of Staphylococcus aureus. Nucleic Acids Res. 16 Feb 2010 (doi:10.1093/nar/gkq058).

59. Bestebroer, J. et al. Staphylococcal superantigen-like 5 binds PSGL-1 and inhibits P-selectin-mediated neutrophil rolling. Blood 109, 2936-2943 (2007).

60. Chavakis, T. et al. Staphylococcus aureus extracellular adherence protein serves as anti-inflammatory factor by inhibiting the recruitment of host leukocytes. Nature Med. 8, 687-693 (2002)

61. Walenkamp, A. M. et al. Staphylococcal superantigenlike 10 inhibits CXCL12-induced human tumor cell migration. Neoplasia 11, 333-344 (2009).

62. Postma, B. et al. Chemotaxis inhibitory protein of Staphylococcus aureus binds specifically to the $\mathrm{C5a}$ and formylated peptide receptor. J. Immunol. 172 6994-7001 (2004).

63. Laursen, N. S. et al. Structural basis for inhibition of complement C5 by the SSL7 protein from Staphylococcus aureus. Proc. Natl Acad. Sci. USA 107, 3681-3686 (2010)

64. Prat, C. et al. A homolog of formyl peptide receptor like 1 (FPRL1) inhibitor from Staphylococcus aureus (FPRL1 inhibitory protein) that inhibits FPRL1 and FPR. J. Immunol. 183, 6569-6578 (2009).

65. Hair, P. S. et al. Clumping factor A interaction with complement factor I increases C3b cleavage on the bacterial surface of Staphylococcus aureus and decreases complement-mediated phagocytosis. Infect. Immun. 78, 1717-1727 (2010).

66. Thammavongsa, V., Kern, J. W., Missiakas, D. M. \& Schneewind, O. Staphylococcus aureus synthesizes adenosine to escape host immune responses. J. Exp. Med. 206, 2417-2427 (2009).
67. Visai, L et al. Immune evasion by Staphylococcus aureus conferred by iron-regulated surface determinant protein IsdH. Microbiology 155, 667-679 (2009).

68. Stephens, D. S., Greenwood, B. ¿ Brandtzaeg, P. Epidemic meningitis, meningococcaemia, and Neisseria meningitidis. Lancet 369, 2196-2210 (2007).

69. Jarva, H., Ram, S., Vogel, U., Blom, A. M. \& Meri, S. Binding of the complement inhibitor C4bp to serogroup B Neisseria meningitidis. J. Immunol. 174 6299-6307 (2005).

70. Schneider, M. C. et al. Functional significance of factor $\mathrm{H}$ binding to Neisseria meningitidis. J. Immunol. 176, 7566-7575 (2006)

71. Madico, G. et al. The meningococcal vaccine candidate GNA1870 binds the complement regulatory protein factor $\mathrm{H}$ and enhances serum resistance. J. Immunol. 177, 501-510 (2006).

72. Lo, H., Tang, C. M. \& Exley, R. M. Mechanisms of avoidance of host immunity by Neisseria meningitidis and its effect on vaccine development. Lancet Infect. Dis. 9, 418-427 (2009).

73. Masignani, V. et al. Vaccination against Neisseria meningitidis using three variants of the lipoprotein GNA1870. J. Exp. Med. 197, 789-799 (2003).

74. Fletcher, L. D. et al. Vaccine potential of the Neisseria meningitidis 2086 lipoprotein. Infect. Immun. 72, 2088-2100 (2004).

75. Seib, K. L. et al. Factor H-binding protein is important for meningococcal survival in human whole blood and serum and in the presence of the antimicrobial peptide LL-37. Infect. Immun. 77, 292-299 (2009).

76. Granoff, D. M., Welsch, J. A. \& Ram, S. Binding of complement factor $\mathrm{H}(\mathrm{fH})$ to Neisseria meningitidis is specific for human $\mathrm{fH}$ and inhibits complement activation by rat and rabbit sera. Infect. Immun. 77, 764-769 (2009).

77. Cantini, F. et al. Solution structure of the immunodominant domain of protective antigen GNA1870 of Neisseria meningitidis. J. Biol. Chem. 281, 7220-7227 (2006).

78. Clantin, B. et al. The crystal structure of filamentous hemagglutinin secretion domain and its implications for the two-partner secretion pathway. Proc. Nat Acad. Sci. USA 101, 6194-6199 (2004).

79. Mascioni, A. et al. Structural basis for the immunogenic properties of the meningococcal vaccine candidate LP2086. J. Biol. Chem. 284, 8738-8746 (2009).

80. Schneider, M. C. et al. Neisseria meningitidis recruits factor $\mathrm{H}$ using protein mimicry of host carbohydrates. Nature 458, 890-893 (2009)

81. Hellerud, B. C. et al. Critical roles of complement and antibodies in host defense mechanisms against Neisseria meningitidis as revealed by human complement genetic deficiencies. Infect. Immun. 78 802-809 (2010)

82. Meri, S., Jordens, M. \& Jarva, H. Microbial complement inhibitors as vaccines. Vaccine 26 (Suppl. 8), 1113-I117 (2008).

83. Beernink, P. T. \& Granoff, D. M. Bactericidal antibody responses induced by meningococcal recombinant chimeric factor $\mathrm{H}$-binding protein vaccines. Infect. Immun. 76, 2568-2575 (2008).

84. Mathew, S. \& Overturf, G. D. Complement and properidin deficiencies in meningococcal disease. Pediatr. Infect. Dis. J. 25, 255-256 (2006).

\section{Acknowledgements}

The authors thank M. Pizza and F. Bagnoli for critical reading of the manuscript. P.G. acknowledges the members of his research group, and financial support from the Council for Chemical Sciences of the Netherlands Organization for Scientific Research and the US National Institutes of Health.

\section{Competing interests statement}

The authors declare competing financial interests: see web version for details.

DATABASES

Entrez Genome Project: http://www.ncbi.nlm.nih.gov/

genomepr:

Bordetella pertussis | Borrelia burgdorferi| group A

Streptococcus | group B Streptococcus | Neisseria meningitidis |

Staphylococcus aureus $\mid$ Streptococcus pneumoniae | Yersinia

enterocolitica

Protein Data Bank: http://www.rcsb.org/pdb/home/home.do 2GOM $\mid 2$ GOX $\mid$ 2KCO $\mid$ 2NOJ $\mid 2$ QFF $\mid$ 2W80 $\mid 2$ WIN $\mid$ KKLS

UniProtKB: http://www.uniprot.or

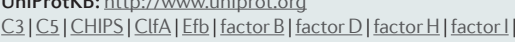

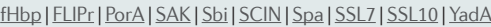

ALL LINKS ARE ACTIVE IN THE ONLINE PDF 